\title{
EQUATIONAL THEORIES WITH A MINORITY POLYNOMIAL
}

\author{
R. PADMANABHAN AND B. WOLK ${ }^{1}$
}

\begin{abstract}
It is known that every finitely based variety of algebras with distributive and permutable congruences is one-based and those admitting a majority polynomial are two-based. In this note we prove two results, one similar to the above and the other in a completely opposite direction: (i) every finitely based variety of algebras of type $\langle 3\rangle$ satisfying the two-thirds minority condition is one-based and (ii) for every natural number $n$, there exists a variety of algebras admitting even a full minority polynomial which is $(n+1)$-based but not $n$-based. An application to the strict consistency of defining relations for semigroups is given.
\end{abstract}

Ever since the appearance of Mal'cev's now classical theorem characterizing the permutability of congruences in a variety, the Mal'cev polynomials have played a steadily increasing role in studying the equational problems of group-like or lattice-like algebras (see, for example, [1], [4], [5] and the thorough survey article [8]). A ternary polynomial $p(x, y, z)$ satisfying the two Mal'cev identities

$$
p(x, x, y)=p(y, x, x)=y
$$

is called a "two-thirds minority" polynomial and it is called a minority polynomial if it further satisfies the third minority condition $p(x, y, x)=y$ as well. In contrast to the minority, there is the concept of a "majority polynomial" satisfying some or all of the identities

$$
p(x, x, y)=p(x, y, x)=p(y, x, x)=x .
$$

Ternary polynomials satisfying the identities (1) or (2) or certain of their combinations have nice universal algebraic significances; thus while (1) is equivalent to the permutability of congruences, (2) is equivalent to the strongest type of congruence distributivity [2]. A particular combination of (1) and (2), viz. two-thirds minority and one-third majority, due to A. F. Pixley [7], is the strongest possible: it gives both the distributive and permutable congruence properties. In [5] it was shown that any finitely based variety of algebras with distributive and permutable congruences is one-based, and those admitting a majority polynomial are two-based. In this note, we prove two results: Every finitely based subvariety of algebras of type $\langle 3\rangle$ satisfying the two-thirds minority identities is one-based and a completely opposite result for finitely based varieties of algebras admitting even a full minority polynomial. Finally, a mixture of majority and minority laws is of interest. The full

Received by the editors June 4, 1980.

1980 Mathematics Subject Classification. Primary 08B05.

${ }^{1}$ The research of both the authors was supported by a grant from NSERC of Canada (\#A8215).

(C) 1981 American Mathematical Society 0002-9939/81/0000-0453/\$02.25 
majority polynomial $(p(x, x, y)=p(x, y, x)=p(y, x, x)=x)$ and the $(1,2)$ combination polynomial $(p(x, x, y)=p(y, x, y)=p(y, x, x)=y)$ were both considered in [5]. The last possibility is the $(2,1)$ combination polynomial $(p(x, x, y)=$ $p(y, x, y)=p(y, x, x)=x)$, but the trivial polynomial $p(x, y, z)=y$ satisfies these identities.

1. Let $\mathbf{M}$ be the variety of all algebras of type $\langle 3\rangle$ in the basic ternary operation satisfying the two-thirds minority condition

$$
p(x, x, y)=p(y, z, z)=y \text {. }
$$

LEMMA 1. In M, the validity of any finite set of identities is equivalent to that of $a$ single identity.

Proof. Let $f=g$ be any identity and let $x$ be a variable not occurring in $f=g$. Then it is clear that $M$ satisfies $f=g$ iff $M$ satisfies $p(f, g, x)=x$. Let $a\left(x_{1}, x_{2}, \ldots, x_{n}\right)=x_{1}$ and $b\left(y_{1}, y_{2}, \ldots, y_{m}\right)=y_{1}$ now be two identities of type $\langle 3\rangle$. It is clear that these two identities together imply

$$
a\left(b\left(y_{11}, y_{12}, \ldots, y_{1 m}\right), b\left(y_{21}, y_{22}, \ldots, y_{2 m}\right), \ldots, b\left(y_{n 1}, y_{n 2}, \ldots, y_{n m}\right)\right)=y_{11} \text {. }
$$

Conversely, if $\mathbf{M}$ satisfies the above identity, then putting $y_{11}=y_{12}=\cdots=$ $y_{1 m}, \ldots, y_{n 1}=y_{n 2}=\cdots=y_{n m}$ and using the idempotency of $p$ we get $a\left(y_{11}, y_{21}, \ldots, y_{n 1}\right)=y_{11}$ and hence $b\left(y_{11}, y_{12}, \ldots, y_{1 m}\right)=y_{11}$. Thus, modulo (1), any two identities, and hence any finite number of identities, can be equivalently expressed by one identity of the form $f\left(x_{0}, \ldots, x_{n}, w\right)=w$ where $w$ is a variable. In what follows, we call the variables $x_{i}$ dummy variables of $f$.

THEOREM 1. Every finitely based subvariety of $\mathbf{M}$ is one-based.

Proof. If $f\left(x_{0}, x_{1}, \ldots, x_{n}, w\right)=w$ is the additional identity which defines the subvariety in question, then it is clear that it does satisfy the identity

$$
f p(f p(x, x, y), f p(z, z, f w), w)=y
$$

where $f u$ is an abbreviation for $f\left(x_{0}, x_{1}, \ldots, x_{n}, u\right)$. Conversely, let us assume the validity of identity (3). We further assume that the set of dummy variables in the outermost $f$ is disjoint from the remaining dummy variables in the inside $f$ 's.

Suppose $x$ abbreviates $f p(a, a, f y)$. Then

$$
f p(x, x, y)=f p(f p(a, a, f y), f p(a, a, f y), y)=f y \quad \text { by (3). }
$$

Now, we see that substituting $f p(a, a, y)$ for $x$ in (3) immediately yields

$$
f p(f y, f p(z, z, f w), w)=y .
$$

From the identities (3) and (4) we get

$$
p(x, x, y)=y
$$

and hence, in particular, $p$ is idempotent. Also, by (4) and (5) we have

$$
f p\left(f y, f^{2} w, w\right)=y \text {. }
$$

Since $p$ is idempotent and $f$ is a $p$-polynomial, by identifying all the dummy variables in " $f y$ " and " $f^{2} w$ " and also $y$ to say $w$, and using the idempotency of $p$ several times we get $f w=w$ and, hence (6) reduces to $p(y, w, w)=y$ and that $p$ is 
a two-thirds minority polynomial and moreover we have the identity $f\left(x_{0}, x_{1}, \ldots, x_{n}, w\right)=w$. This completes the proof of the theorem.

COROLlaRY 1. The property of a ternary polynomial $p(x, y, z)$ satisfying all the three minority identities

$$
p(x, x, y)=p(y, x, x)=p(x, y, x)=y
$$

can be expressed by a single identity.

The above result follows, of course, from Theorem 1 by simply taking $p\left(x_{0}, w, x_{1}\right)$ for $f w$ in the main identity (3) of that theorem. However, the following simple identity will do:

$$
p(p(x, x, y), p(z, w, z), w)=y .
$$

The minority polynomial is conceptually as nice and simple as, say, the majority polynomial. Both are expressible by a single identity (see Lemma 3 of [5]), and also, in presence of a majority polynomial, there is available a general method of reducing the validity of two identities to that of one (this result is due to $K$. A. Baker [1], see also Lemma 1 of [5]). But such a general method of reducing a set of $n$ identities to $n-1$ identities in general algebras does not exist in the presence of even a full minority polynomial.

For $n\rangle 1$, let $K_{n}$ be the variety of all algebras of type $\tau=\langle 3,1, \ldots, 1\rangle$ with the fundamental operations $p(x, y, z), h_{1}(x), \ldots, h_{n}(x)$ satisfying the identities

$$
\begin{aligned}
p(x, x, y) & =p(x, y, x)=p(y, x, x)=y, \\
h_{1}(x) & =h_{2}(x)=\cdots=h_{n}(x)=x .
\end{aligned}
$$

THEOREM 2. This variety $K_{n}$ is $(n+1)$-based but not $n$-based.

To show $K_{n}$ is not $n$-based, we use $2^{n+1}$ algebras of type $\tau$. Let $\langle F ;+, *, 0,1\rangle$ be the two-element field, and let $V=F^{n+1}$ be an $(n+1)$-dimensional vector space over $F$. For each $v \in V, v=\left\langle v_{0}, v_{1}, \ldots, v_{n}\right\rangle$, define an algebra $A_{v}$ of type $\tau$ over $F$ by interpreting the operations of $\tau$ on $F$ as follows:

$$
\begin{aligned}
\left.p\right|_{A_{\mathrm{v}}}(x, y, z) & =x+y+z+v_{0}, \\
\left.h_{i}\right|_{A_{\mathrm{v}}}(x) & =x+v_{i} \text { for } i=1,2, \ldots, n .
\end{aligned}
$$

Then $A_{v} \in K_{n}$ iff $v=\langle 0,0, \ldots, 0\rangle=0$.

LEMMA 2. Let $r=s$ be an identity of $K_{n}$. Then $\left\{v \mid r=s\right.$ holds in $\left.A_{v}\right\}$ is a subspace of $V$ of dimension $n$.

Proof. (For example, $p\left(x, h_{2}(y), y\right)=x$ holds in $A_{v}$ iff $v_{0}+v_{2}=0$.) Note $r$ and $s$ are polynomials in the operations of $\tau$, and the identity $r=s$ holds in $A_{0}$. Let $r_{v}$ be the polynomial over $F$ produced by the interpretation (10) of $\tau$ in $A_{v}$, and define $s_{v}$ similarly. Then the various $r_{v}$ 's differ only in their constant terms. To describe this variation, define $h_{0}=p$, and let $\mathbf{r}=\left\langle\mathbf{r}_{0}, \mathbf{r}_{1}, \mathbf{r}_{2}, \ldots, \mathbf{r}_{n}\right\rangle$ be a vector of length $n+1$, where $r_{i}=$ the number of occurrences of the operation $h_{i}$ in the polynomial $r$. Let $\mathbf{r}^{\prime}$ be the modulo 2 reduction of $\mathbf{r}$. So $\mathbf{r}^{\prime}$ is a vector in $V$. Then it is easy to see that $r_{v}=r_{0}+\left(\mathbf{r}^{\prime} \cdot v\right)$, where $\cdot$ denotes the usual dot product of vectors in $V$. Similarly, $s_{v}=s_{0}+\left(s^{\prime} \cdot v\right)$. 
Now $r_{0}=s_{0}$, since $r=s$ holds in $A_{0}$. So $r_{v}-s_{v}=\left(r^{\prime}-s^{\prime}\right) \cdot v$, using obvious meanings for "subtraction". This shows that $r=s$ holds in $A_{v}$ iff $r_{v}=s_{v}$ iff $v$ is orthogonal to the vector $\mathbf{r}^{\prime}-\mathbf{s}^{\prime}$. Since the set of all Such vectors $v$ forms a subspace of codimension 1, the lemma is proved.

Proof of Theorem 2. Corollary 1 shows that $K_{n}$ can be defined by the $n+1$ identities of (8) and (9). So $K_{n}$ is at most $(n+1)$-based. If $\left\{r_{i}=s_{i} \mid i=1,2, \ldots, n\right\}$ are any $n$ identities of $K_{n}$, let $V_{i}=\left\{v \in V \mid r_{i}=s_{i}\right.$ holds in $\left.A_{v}\right\}$. By Lemma 2, each $V_{i}$ is a subspace of codimension 1 . The intersection of $n$ such subspaces has codimension at most $n$, and is therefore a nonzero subspace. This means that all $n$ identities are true in some $A_{v}$, with $v \neq 0$. Since this $A_{v}$ is not in $K_{n}$, these $n$ identities do not define $K_{n}$. So $K_{n}$ is not $n$-based.

2. An application to semigroups. Let $X=\left\{x_{0}, x_{1}, \ldots, x_{n}\right\}$ be an alphabet and let $R$ be a set of defining relations over $X . R$ is said to be strictly consistent if there is at least one nontrivial semigroup generated by $X$ and satisfying the defining relations in $R$.

Corollary 2. Let $X=\left\{x_{0}, x_{1}, \ldots, x_{n}\right\}$ and $R$ any set of defining relations over $X$. If $|R|<n+1$ then $R$ is always strictly consistent.

Proof. Let $R=\left\{r_{i}\left(x_{0}, \ldots, x_{n}\right)=s_{i}\left(x_{0}, \ldots, x_{n}\right) \mid i=1, \ldots, k, k<n+1\right\}$ be not strictly consistent. Let us formally represent the generators $x_{i}$ as mappings of $F$, the two-element field, thus

$$
x_{i}: F \rightarrow F \quad \text { where } x_{i}(y)=h_{i}(y)
$$

Now each semigroup word $r\left(x_{0}, x_{1}, \ldots, x_{n}\right)$ corresponds to a mapping $r\left(h_{0}, h_{1}, \ldots, h_{n}\right): F \rightarrow F$ computed as per the composition of the word $r$. For each $i \in\{1,2, \ldots, k\}$, define

$$
S_{i}=\left\{v \mid A_{v} \vDash r_{i}=s_{i}\right\}
$$

where now $r_{i}\left(h_{0}, h_{1}, \ldots, h_{n}\right)=s_{i}\left(h_{0}, h_{1}, \ldots, h_{n}\right)$ is an identity in the language of $\tau$. By the lemma, each $S_{i}$ is a subspace of $V$ of dimension $n$. Hence,

$$
\bigcap_{i=1}^{k} S_{i}=\left\{v \mid A_{v} \vDash r_{i}=s_{i} \text { for all } i \in\{1,2, \ldots, k\}\right\}
$$

is a subspace of dimension $n+1-k$ and the collection of defining relations $R=\left\{r_{i}=s_{i} \mid i=1,2, \ldots, k\right\}$ will be strong enough not to be strictly consistent iff $\cap_{i=1}^{k} S_{i}=\{0\}$, the null space. This can happen iff $n+1-k=0$ or $k=n+1$ but we are given that $k<n+1$. Hence, for any $R$ with $|R|<n+1$, there exists a nontrivial semigroup generated by $n$ elements and satisfying all the $k$ defining relations in $R$. Actually, this semigroup-represented as mappings-can easily be computed by noting that $A_{v} \vDash r=s$ iff $v \cdot(\mathbf{r}-\mathbf{s})=0$. This number $|X|-|R|$, known as the deficiency number, has been widely studied in group theory (see p. 93 of [3]). 


\section{REFERENCES}

1. K. A. Baker, Finite equational bases for finite algebras in a congruence-distributive equational class, Adv. in Math. 24 (1977), 207-243.

2. B. Jonsson, Algebras whose congruence lattices are distributive, Math. Scand. 21 (1967), 110-121.

3. R. C. Lyndon and P. E. Schupp, Combinational group theory, Springer-Verlag, New York, 1977.

4. R. N. McKenzie, On spectra and the negative solution of the decision problem for identities having a finite nontrivial model, J. Symbolic Logic 40 (1975), 186-196.

5. R. Padmanabhan and R. Quackenbush, Equational theories of algebras with distributive congruences, Proc. Amer. Math. Soc. 41 (1973), 373-377.

6. R. Padmanabhan, Equational theory of algebras with a majority polynomial, Algebra Universalis 7 (1977), 273-275.

7. A. F. Pixley, Distributivity and permutability of congruence relations in equational classes of algebras, Proc. Amer. Math. Soc. 14 (1963), 105-109.

8. W. Taylor, Equational logic, Appendix 4 in G. Grätzer, Universal algebra, 2nd ed., Springer-Verlag, New York, 1979.

Department of Mathematics, University of Manttoba, Winnipeg, Manitoba, Canada R3T 2N2 